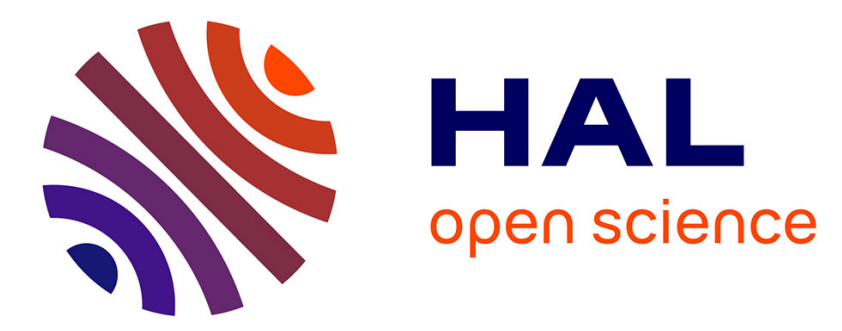

\title{
A brief history of first-order shear-deformable beam and plate models
}

Noël Challamel, Isaac Elishakoff

\section{To cite this version:}

Noël Challamel, Isaac Elishakoff. A brief history of first-order shear-deformable beam and plate models. Mechanics Research Communications, 2019, 102, pp.103389 10.1016/j.mechrescom.2019.06.005 . hal-03488560

\section{HAL Id: hal-03488560 \\ https://hal.science/hal-03488560}

Submitted on 21 Dec 2021

HAL is a multi-disciplinary open access archive for the deposit and dissemination of scientific research documents, whether they are published or not. The documents may come from teaching and research institutions in France or abroad, or from public or private research centers.
L'archive ouverte pluridisciplinaire HAL, est destinée au dépôt et à la diffusion de documents scientifiques de niveau recherche, publiés ou non, émanant des établissements d'enseignement et de recherche français ou étrangers, des laboratoires publics ou privés.

\section{다)(1) $(5$}

Distributed under a Creative Commons Attribution - NonCommercial| 4.0 International 


\section{A Brief History of First-Order Shear- Deformable Beam and Plate Models}

\author{
Noël Challamel ${ }^{1}$ and Isaac Elishakoff ${ }^{2}$ \\ ${ }^{1}$ Université de Bretagne Sud \\ Institut de Recherche Dupuy de Lôme (IRDL) \\ UMR CNRS 6027 \\ Centre de Recherche \\ Rue de Saint Maudé - BP 92116 \\ 56321 Lorient cedex - FRANCE \\ Email : noel.challamel@univ-ubs.fr \\ ${ }^{2}$ Florida Atlantic University, \\ Boca Raton, FL 33431-0991 USA \\ Phone: (407) 367-2729 - \\ Email : elishako@fau.edu
}

\begin{abstract}
This paper presents a brief history of beam and plate models in elasticity, that take into account the rotary inertia and shear contribution. We show that Bresse (1859) already in the mid XIX ${ }^{\text {th }}$ century rigorously derived the set of equations for curved shear and axially extensible beams in dynamics, although without shear correction factor. When restricted to straight beams, Bresse (1859) obtained a two-field beam kinematics composed of independent deflection and rotation variables. These equations have been generalized by Timoshenko (1913; 1916; $1920 ; 1921 ; 1922)$, without direct reference to the works of Bresse, by a slight modification of the shear stiffness, that incorporates a shear correction factor generally different from unity. The calibration of the shear correction factor with respect to threedimensional elasticity solutions has been elaborated jointly by Ehrenfest and Timoshenko in the second decade of the $\mathrm{XX}^{\text {th }}$ century (the calibration of the shear correction factor for rectangular cross section has been finalized by Timoshenko in 1922, even if the basic results were available in his letter dated from 1913, in collaboration with Ehrenfest). Whereas Bresse-Timoshenko-Ehrenfest equations have been elaborated mainly during more than half a century between 1859 and 1922 (even if researches on shear beam theories were still active), the Uflyand-Mindlin plate theory has been built in a more compact period, mainly between 1944 and 1951. Whereas Reissner (1944, 1945) developed a static (or stress-based) formulation, the kinematic theory of Uflyand-Mindlin plate model has been first elaborated by Bolle (1947) and Hencky (1947) for static setting, before the full generalization to dynamics by Uflyand a year later, in 1948, and its complete variational derivation by Mindlin (1951). A lot of efforts were spent since almost half of the
\end{abstract}

century to calibrate the shear correction factor and to enrich the kinematics of Uflyand-Mindlin plate theory. It is quite surprising that the shear correction factors of both the Bresse-Timoshenko-Ehrenfest beam model and its Uflyand-Mindlin plate analogy were already implicitly available in the paper of Timoshenko (1922), performed, in his own testimony with Ehrenfest.

Key-words: Beam theory; Plate theory; History of structural mechanics; Bresse-Timoshenko-Ehrenfest beam theory; Uflyand-Mindlin plate theory; Elasticity; Shear effects

\section{Introduction}

The effect of introducing shear contribution in the theory of elastic structures is of primary importance for engineering design, both in statics and in dynamics. A beam theory, which already included the bending and the shear effects through two independent kinematic fields for a straight beam, namely the displacement and the rotation variables, was developed in the mid XIX $^{\text {th }}$ century by Bresse (Bresse, 1859). Bresse (1859) also considered the rotary effect separately. Timoshenko, later, at the beginning of the $\mathrm{XX}^{\text {th }}$ century extended Bresse's theory with the introduction of a shear correction factor, in a fully consistent beam framework (Timoshenko, 1913; Timoshenko, 1916; Timoshenko, 1920; Timoshenko, 1921; Timoshenko, 1922). Even if Timoshenko in his book and in his papers dated from the beginning of the $\mathrm{XX}^{\text {th }}$ century did not mention Bresse's work for elaborating his shear beam theory, he only pointed out the contribution of Bresse in his book on history of structural mechanics (Timoshenko, 1953, page 151), where he recognized that Bresse "was the first to take rotatory inertia of the elements of the bar into consideration." In fact, Bresse (1859)'s contribution is greatly more than including the rotary effect: he developed the fully consistent bending-shear theory based on independent kinematic variables, namely the deflection, the axial displacement and the rotation for the in-plane behavior of curved beam elements. This major contribution of Bresse in establishing a fully consistent first-order shear beam theory was acknowledged in the literature (see for instance Mindlin and Deresiewicz, 1954; Bert, 1981; Abramovich and Elishakoff, 1987; Mindlin, 2006; Elishakoff, 2010 or more recently Elishakoff, 2019a). The shear correction factor, introduced by Engesser (1891) and Föppl (1897) was implicitly equal to unity in Bresse's theory. The following quotation from Elishakoff's (2019-a) book appears to be instructive: "According to the monograph by Artobolevsky, Bobrovnitsky and Genkin (1979, pp. 142-143), in a free translation from the Russian, in 
the works of scientists of the 18th and 19th centuries the taking into account of shear deformation was mainly focused on static bending. Thus, in $1856 \mathrm{~B}$. St. Venant gave a rigorous solution to the static problem of the bending of a cantilever by a concentrated force applied at the tip. He showed that the distribution of the shear stresses across the height was a quadratic parabola. In dynamic setting the shear was accounted for, for the first time by Bresse (1859), Bresse's equations describe transversallongitudinal vibrations of the curved beams, the central line of which lies in one plane, and in addition to the shear takes into account the rotary inertia of the cross-section. For the straight beam, Bresse's equations are split into an equation for longitudinal waves and the equation for transverse vibrations coinciding with Timoshenko's equations, in which the shear coefficient equals unity. "

Starting from the inception of BresseTimoshenko-Ehrenfest's beam theory, this brief history devoted to the introduction of shear effects in structural mechanics presents a new reading of what is sometimes called the Timoshenko beam theory, and its plate generalization usually labelled as the Mindlin plate model. Only first-order shear beam or plate theories will be presented. Higher-order shear beam or plate models, where higher-order kinematic fields are considered are not treated in this historical synthesis. The reader can refer to the books of Elishakoff and Irretier (1987), Wang et al (2000), Reddy (2004) or Carerra et al (2011) for a presentation of higher-order shear structural members (see also the historical paper of Reissner, 1985 on the presentation of various plate theories including higher-order shear plate theories).

\section{Beam theory}

Bresse (1859) during the mid XIX ${ }^{\text {th }}$ century was the first who developed a fully consistent shear beam theory for curved rods. Bresse's contribution is highlighted and commented in the first part of this historical analysis.

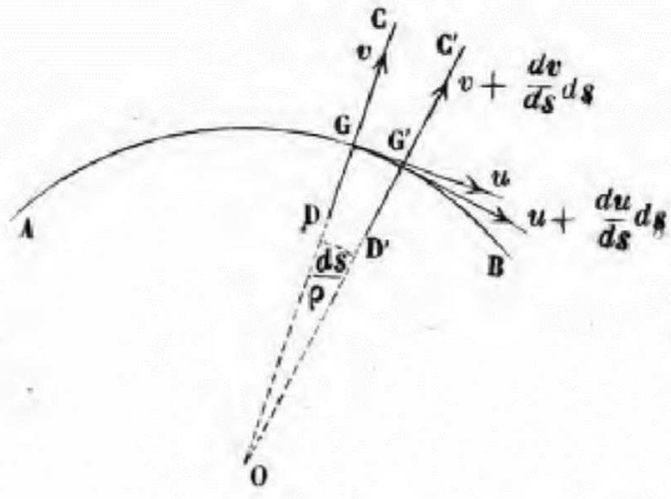

Figure 1 - Definition of the curved element and the displacement field in the deformed configuration (from Fig. 32 page 123 of Bresse, 1859)

In Figure 1 (taken from the book of Bresse, 1859), $u$ is the axial displacement, $v$ is the deflection in the orthogonal direction, $s$ is the curvilinear abscissa, $d s$ is the differential curvilinear abscissa and $\rho$ is the curvature radius. Bresse (1859) also used the following notation: $\theta$ is the independent rotation, $t$ is the time, $\Omega$ is the cross sectional area, $g$ is the acceleration of gravity, and $\pi$ is the volumetric weight (equal to the product between the mass density and $g$ ). It is assumed that the beam is composed of an isotropic linear elastic material, with $G$ as the shear modulus, and $E$ the Young modulus. $k=G / E$ is the ratio between the shear modulus and the Young modulus. Bresse (1859) first introduced the rotary inertia, proportional to the rotational acceleration. $d^{2} \theta / d t^{2}$ (as highlighted in Figure 2), in a fully consistent beam theory where the rotation is considered as an independent degree of freedom.

\footnotetext{
La masse totale du prisme $\operatorname{CDC}^{\prime} D^{\prime}$ est $\frac{\pi}{g} \Omega d s$; l'accélératión de son centre de gravité a pour composantes $\frac{d^{2} u}{d t^{2}}$ et $\frac{d^{2} v}{d t^{2}}$; la résultante de translation des forces d'inertie de cette masse aura donc pour composantes, parallèlement à $u$ et à $v$, les forces $-\frac{\Pi}{g} \Omega d s \frac{d^{2} u}{d t^{2}},-\frac{\Pi}{g} \Omega d s \frac{d^{2} v}{d t^{2}}$. D'un autre côté, $\operatorname{CDC}^{\prime} \mathbf{D}^{\prime}$ tourne autour de son centre de gravité avec une vitesse angulaire $\frac{d \theta}{d t}$ et une accélération angulaire $\frac{d^{2} 0}{d t^{2}}$, d'où résulte un couple d'inertie ayant pour intensité le produit du moment d'inertie par $-\frac{d^{2} \theta}{d t^{2}}, c^{\prime}$ est-à-dire $-\frac{\Pi}{g} \Omega d s \cdot r^{2} \frac{d^{2} \theta}{d t^{2}}$.
}

Figure 2 - Definition of the curved element and the displacement field in the deformed configuration (from page 124 of Bresse, 1859) 
which can be translated as "The total mass of the prismatic element $C D C^{\prime} D$ ' is $\pi / g \Omega d s$; the acceleration of its center of gravity has two components namely $d^{2} u / d t^{2}$ and $d^{2} v / d t^{2}$; the translational inertia forces have then two components parallel to the axis associated with the displacements $u$ and $v$, i.e. $-\pi / g \Omega d s d^{2} u / d t^{2}$ and $-\pi / g \Omega d s d^{2} v / d t^{2}$. Furthermore, the prismatic element $C D C^{\prime} D$ ' turns around its center of gravity with the angular rate $d \theta / d t$ and its angular acceleration $d^{2} \theta / d t^{2}$, thus leading to a resulted torque which is the product between the rotary inertia by $-d^{2} \theta / d t^{2}$, i.e. $-\pi / g \Omega d s r^{2} d^{2} u / d t^{2}$ ”.

Then, on page 126, Bresse (1859) expresses the internal forces and moment contribution, first from the balance of internal force along the axial direction:

Force parallèle à $\mathbf{G} u$ :

$$
\mathbf{E} \Omega d s\left[\frac{d}{d s}\left(\frac{d u}{d s}+\frac{v}{\rho}\right)+\frac{k}{\rho}\left(\frac{d v}{d s}+0-\frac{u}{\rho}\right)\right]
$$

Figure 3 - Expression of the axial forces along the differential element $d s$ (from page 126 of Bresse, 1859)

The balance of internal force along the orthogonal vertical direction is given by:

Force parallèle à $\mathbf{G} v$ :

$$
\mathrm{E} \Omega d s\left[k \frac{d}{d s}\left(\frac{d v}{d s}+\theta-\frac{u}{\rho}\right)-\frac{1}{\rho}\left(\frac{d u}{d s}+\frac{v}{\rho}\right)\right]
$$

Figure 4 - Expression of the vertical forces along the differential element $d s$ (from page 126 of Bresse, 1859)

The balance of internal moment is defined from:

Moment total par rapport au centre de gravité de l'élén évalué dans le sens de la rotation $\theta$ :

$$
\mathbf{E} \Omega d s\left[r^{2} \frac{d^{2} \theta}{d s^{2}}-k\left(\frac{d v}{d s}+\theta-\frac{u}{\rho}\right)\right] .
$$

Figure 5 - Expression of the moment along the differential element $d s$ (from page 126 of Bresse, 1859)

The total balance of internal forces and moment including the inertia contributions, is formulated in Figure 6 (from the book of Bresse, 1859), which are the dynamic balance equations of the curved elastic beam (with bending, shear and axial rigidity) incorporating shear and rotary effects:

Par suite on trouvera pour les équations du mouvement de l'élément prismatique considéré :

$$
\text { (1) }\left\{\begin{array}{l}
\frac{d^{2} u}{d t^{2}}=\mathrm{R}+\frac{\mathrm{E} g}{\mathrm{n}}\left[\frac{d}{d s}\left(\frac{d u}{d s}+\frac{v}{\rho}\right)+\frac{k}{\rho}\left(\frac{d v}{d s}+\theta-\frac{u}{\rho}\right)\right] ; \\
\frac{d^{2} v}{d t^{2}}=\mathrm{T}+\frac{\mathrm{E} g}{\mathrm{n}}\left[h \frac{d}{d s}\left(\frac{d v}{d s}+\theta-\frac{u}{\rho}\right)-\frac{1}{\rho}\left(\frac{d u}{d s}+\frac{v}{\rho}\right)\right] ; \\
r^{2} \frac{d^{2} \theta}{d t^{2}}=\mathrm{M}+\frac{\mathrm{Eg}}{\mathrm{H}}\left[r^{2} \frac{d^{2} \theta}{d s^{2}}-k\left(\frac{d v}{d s}+\theta-\frac{u}{\rho}\right)\right] .
\end{array}\right.
$$

Figure 6 - Bresse's equations of the dynamics of a curved beam incorporating shear and rotary effects - Eq. (1) Page 126 of Bresse (1859)

$(R, T, M)$ are the normalized distributed axial, lateral and external couples. Neglecting these distributed forces, these governing equations can be reformulated with modern notations as:

$$
\begin{gathered}
\left\{\begin{array}{c}
\rho A \frac{\partial^{2} u}{\partial t^{2}}=\frac{\partial N}{\partial s}+\frac{V}{R} \\
\rho A \frac{\partial^{2} v}{\partial t^{2}}=-\frac{\partial V}{\partial s}+\frac{N}{R} \\
\rho I \frac{\partial^{2} \psi}{\partial t^{2}}=\frac{\partial M}{\partial s}-V
\end{array}\right. \\
\left\{\begin{array}{c}
N=E A\left(\frac{\partial u}{\partial s}-\frac{v}{R}\right) \\
V=-G A\left(\frac{\partial v}{\partial s}-\psi+\frac{u}{R}\right) \\
M=E I \frac{\partial \psi}{\partial s}
\end{array}\right.
\end{gathered}
$$

with

with the new notations, $u$ is the axial displacement, $v$ is the deflection (counted in the opposite direction, as compared to Bresse's convention), $\psi=\theta$ is the independent rotation. The curvature radius is equal to $R$, the mass density is denoted by $\rho, N$ is the normal force, $V$ is the shear force, $M$ is the bending moment, $A$ is the cross section area and $I$ is the moment of inertia. 
Eq. (1) are the linearized equations of the curved Bresse element, whose expression in a geometrically exact framework is available for instance in the book of Luongo and Zulli (2013). The equations of curved Bresse-Timoshenko elements may be also derived from asymptotic analysis, starting from threedimensional elasticity, as shown by Berdichevskii and Starosel'skii (1983). Berdichevskii and Starosel'skii (1983) also gave some variational arguments for general curved Bresse-Timoshenko rods, including possible coupling between the elongation and flexure, flexure with torsion and shear contributions. Analytical frequency solutions for Bresse-Timoshenko circular rings are calculated by Rao and Sundararajan (1969) in presence of inextensibility, and by Issa et al (1987) with the inextensibility assumption relaxed. Coupled axial and rotational inertia may be considered as well, with the incorporation of additional inertia terms in the dynamic balance equation, as shown by Morley (1961), Irie et al (1983) or Kang et al (1995). The dynamics of curved shear beam elements is investigated in standard textbooks such as the books of Borg and Gennaro (1969), Henrych (1981) or Rao (2007).

For straight beams, i.e. when $R$ tends towards an infinite value, the axial and shear-bending wave equations Eq. (1) are uncoupled, and may be presented in the following form:

$$
\left\{\begin{array}{c}
\rho A \frac{\partial^{2} u}{\partial t^{2}}=E A \frac{\partial^{2} u}{\partial x^{2}} \\
\rho A \frac{\partial^{2} v}{\partial t^{2}}=G A\left(\frac{\partial^{2} v}{\partial x^{2}}-\frac{\partial \psi}{\partial x}\right) \\
\rho I \frac{\partial^{2} \psi}{\partial t^{2}}=E I \frac{\partial^{2} \psi}{\partial x^{2}}+G A\left(\frac{\partial v}{\partial x}-\psi\right)
\end{array}\right.
$$

As we will mainly focus on the bending-shear coupling, Bresse's coupled equations can finally be presented for straight beams as:

$$
\left\{\begin{array}{c}
-G A\left(v^{\prime \prime}-\psi^{\prime}\right)+\varrho A \ddot{v}=0 \\
-E I \psi^{\prime \prime}-G A\left(v^{\prime}-\psi\right)+\varrho I \ddot{\psi}=0
\end{array}\right.
$$

where the prime denotes spatial derivation with respect to the horizontal axis whereas the dot is associated to the time derivative.

These equations, as elaborated by Bresse (1859) do not contain shear stiffness corrections. In fact, Bresse (1859) did not mention the concept of shear correction factor, which has been first introduced much later, namely by Engesser (1891) and Föppl (1897). This coefficient implicitly takes into account the fact that the shear strain is not uniform in general, over the beam's cross section. Engesser (1891) and Föppl (1897) calculated in statics and from energy arguments, the shear correction factor for a rectangular cross section as:

$$
\kappa=\frac{5}{6}
$$

Timoshenko (1913, 1916, 1920, 1921, 1922) then generalized Bresse equations by introducing such a shear factor $\kappa$ in correction of the shear stiffness:

$$
\left\{\begin{array}{c}
-\kappa G A\left(v^{\prime \prime}-\psi^{\prime}\right)+\varrho A \ddot{v}=0 \\
-E I \psi^{\prime \prime}-\kappa G A\left(v^{\prime}-\psi\right)+\varrho I \ddot{\psi}=0
\end{array}\right.
$$

Timoshenko equations Eq. (5) thus include Bresse equations for a shear correction factor formally set equal to unity $\kappa=1$. This value $\kappa=1$ (implicitly contained in the theory of Bresse) may be also used for Cosserat beam theory (which merges with Bresse theory in its linearized form), as shown by Rubin (2003). From Eq. (5), it is possible to present a single bending-shear wave equation valid for BresseTimoshenko-Ehrenfest beam:

$$
E I \frac{\partial^{4} v}{\partial x^{4}}-\rho I\left(1+\frac{E}{\kappa G}\right) \frac{\partial^{4} v}{\partial x^{2} \partial t^{2}}+\rho A \frac{\partial^{2} v}{\partial t^{2}}+\frac{\rho^{2} I}{\kappa G} \frac{\partial^{4} v}{\partial t^{4}}=0
$$

Eq. (6) is a linear fourth-order partial differential equation both in space and in time. This partial differential equation was already available in the letter that Timoshenko sent to Ehrenfest in 1913 (see Figure 6 with different notations for each component).

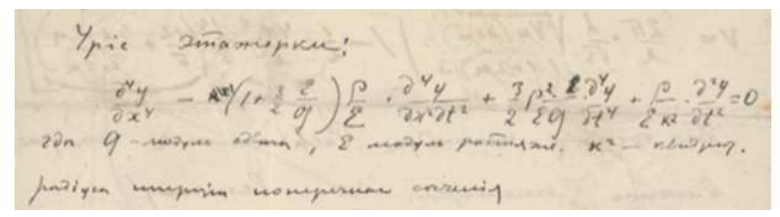

Figure 6 - The first appearance of the full BresseTimoshenko-Ehrenfest theory in a private correspondence between Timoshenko and Ehrenfest - Timoshenko (1913) - see also Elishakoff (2019-a) for a complete analysis of this correspondence

The first appearance of this partial differential equation in a public document is the edition of the book of Timoshenko in 1916 (in Russian) where Eq. (178) on page 207 of Timoshenko (1916) coincides with Eq. (6) of the present paper (see Figure 7): 


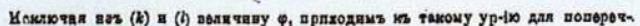
Kactio 4 an 893

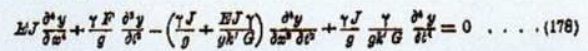

Figure 7 - Eq. (178) on page 207 of Timoshenko (1916); $k^{\prime}$ in this equation is the shear correction factor denoted by $\kappa$ in this paper

Four years later, Timoshenko (1920) published his first paper on this shear beam theory in a Croatian journal (in English), where again this partial differential equation is available in a different form (see Figure 8):

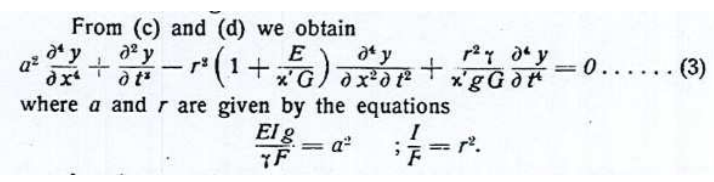

Figure 8 - Eq. (3) of Timoshenko (1920); $\chi^{\prime}$ in this equation is the shear correction factor denoted by $\kappa$ in this paper

Timoshenko decided to publish two closely related papers in 1921 and in 1922 in the well reputed journal Philosophical Magazine (Timoshenko, 1921; Timoshenko, 1922). The main results however were available in the paper already published in 1920, as also commented by Elishakoff (2019-a).

The reader is reported to the extensive bibliographical study of Elishakoff (2019-b) for a detailed analysis of Timoshenko's life, especially during this crucial period of the end of the second decade of the $\mathrm{XX}^{\text {th }}$ century, where Timoshenko decided to emigrate from Ukraine and Russia (see also Elishakoff, 2019-c).

Timoshenko in his papers dated from the beginning of the last century recommended several values for the shear correction factor. He used $\kappa=\frac{5}{6}$ in Timoshenko (1920) (following Engesser and Föppl's recommendation) and $\kappa=\frac{2}{3}$ in Timoshenko (1916; 1921). In his paper dated from 1922 (Timoshenko, 1922), Timoshenko calibrated the shear correction factor for an elastic beam composed of rectangular cross section, from the exact twodimensional wave length solution, also labelled as Rayleigh-Lamb equation (Rayleigh, 1889; Lamb, 1921 - see also Mindlin, 1951). Starting from the solution of plane wave based on plane strain assumptions, Timoshenko and Ehrenfest (Timoshenko, 1922) jointly obtained a transcendental frequency equation based on hyperbolic tangent tanh function (see also Rayleigh, 1889; Lamb, 1921) which is asymptotically expanded, with respect to the small relative depth ratio of the beam, for an explicit comparison with the Bresse-Timoshenko-Ehrenfest beam solution (see also Elishakoff, 2019-a). The comparison of the frequency equation of each model (the twodimensional elasticity solution and the beam solution) gives:

$$
\begin{gathered}
1+\frac{E}{\kappa G}=\frac{3 \lambda^{\prime}+2 \mu^{\prime}}{\lambda^{\prime}+2 \mu^{\prime}}+\frac{12}{5} \quad v^{\prime}=\frac{v}{1+v}
\end{gathered}
$$

where $v$ is the Poisson's ratio valid for a plane stress analysis and $v^{\prime}$ can be used for a plane strain analysis. Eq. (7) can be equivalently reformulated as:

$$
1+\frac{2(1+v)}{\kappa}=\frac{1+v^{\prime}}{1-v^{\prime}}+\frac{12}{5}=1+2 v+\frac{12}{5}
$$

As pointed out by Timoshenko in his paper dated from 1922 (and in his book dated from 1916), the frequency equation was elaborated by Ehrenfest and himself. Timoshenko addressed his letter in 1913 to Ehrenfest on the elaboration of the so-called BresseTimoshenko-Ehrenfest theory (Timoshenko, 1913 see also the detailed historical analysis of Elishakoff, 2019-a on this point). $\kappa$, the shear correction factor is then identified from this asymptotic analysis valid for a rectangular cross section and based on the plane stress assumption for a valid comparison with the beam theory:

$$
\kappa=\frac{5(1+v)}{6+5 v}
$$

Kaneko (1975) also commented this value, and mentioned that already Timoshenko (1922) implicitly used this formulae. This value is found by Stephen (1981) by comparing the response of the simply supported Bresse-Timoshenko beam with the one issued of a two-dimensional elasticity solution also based on plane stress assumption. The same value was obtained by Elishakoff et al (2015) from an asymptotic expansion of the three-dimensional displacement field with respect to the dimensionless depth ratio with plane stress assumptions. Elishakoff et al (2015) also derived a truncated BresseTimoshenko model from this asymptotic expansion:

$$
E I \frac{\partial^{4} v}{\partial x^{4}}-\rho I\left(1+\frac{E}{\kappa G}\right) \frac{\partial^{4} v}{\partial x^{2} \partial t^{2}}+\rho A \frac{\partial^{2} v}{\partial t^{2}}=0
$$


This asymptotically-based model differs from the original Bresse-Timoshenko-Ehrenfest model from the fourth-order time derivative which has been removed in Eq. (6). Such a truncated BresseTimoshenko-Ehrenfest model was initially proposed by Elishakoff and Lubliner (1985) (see also Elishakoff and Livshits, 1989).

Bresse-Timoshenko-Ehrenfest equations can be formulated in statics in presence of distributed loading $p$ as (see for instance Timoshenko, by differentiating two times Eq. (106) on page 171 of Timoshenko, 1940):

$$
E I \frac{\partial^{4} v}{\partial x^{4}}=p-\frac{E I}{\kappa G A} \frac{\partial^{2} p}{\partial x^{2}}
$$

Eq. (11) is obtained from the static governing equations applied to the Bresse-Timoshenko beam theory:

$$
\left\{\begin{array}{c}
-\kappa G A\left(v^{\prime \prime}-\psi^{\prime}\right)-p=0 \\
-E I \psi^{\prime \prime}-\kappa G A\left(v^{\prime}-\psi\right)=0
\end{array}\right.
$$

The generalization of Bresse-Timoshenko-Ehrenfest beam equations for statics and dynamics is written by combining Eq. (5) and Eq. (12), thus leading to:

$$
\left\{\begin{array}{c}
-\kappa G A\left(v^{\prime \prime}-\psi^{\prime}\right)+\varrho A \ddot{v}-p=0 \\
-E I \psi^{\prime \prime}-\kappa G A\left(v^{\prime}-\psi\right)+\varrho I \ddot{\psi}=0
\end{array}\right.
$$

which can be reformulated in a single partial differential equation:

$$
\begin{aligned}
& E I \frac{\partial^{4} v}{\partial x^{4}}-\rho I\left(1+\frac{E}{\kappa G}\right) \frac{\partial^{4} v}{\partial x^{2} \partial t^{2}}+\rho A \frac{\partial^{2} v}{\partial t^{2}} \\
& +\frac{\rho^{2} I}{\kappa G} \frac{\partial^{4} v}{\partial t^{4}}=p-\frac{E I}{\kappa G A} \frac{\partial^{2} p}{\partial x^{2}}+\frac{\rho I}{\kappa G A} \frac{\partial^{2} p}{\partial t^{2}}
\end{aligned}
$$

\section{Plate theory}

The generalization of Bresse-Timoshenko-Ehrenfest beam theory which incorporates shear effect to plate has been developed in the 40's (see the historical footnotes mentioned by Timoshenko and Woinowsky-Krieger, 1959 or the more extensive historical presentation of Reissner, 1985). Reissner (1944, 1945) presented a stress-based theory, which accounts for shear effect in statics. Reissner (1944, 1945) obtained a partial differential equation for the deflection of an isotropic plate, which is written (see Figure 9):

$$
D \Delta^{2} v=p-\frac{h^{2}(2-v)}{10(1-v)} \Delta p
$$

with the bending rigidity of the plate which is classically equal to $D=E \frac{h^{3}}{12\left(1-v^{2}\right)}$ for a plate of depth $h$.

$$
D \Delta \Delta w=p-\frac{2-\nu}{10(1-\nu)} h^{2} \Delta p
$$

Clearly, the second term on the right of equation (17) 18 insignificant unless $p$ changes appreciably within distances of the order of the plate thickness.

Figure 9 - Eq. (17) of Reissner (1944); The second term at the right-hand side of Eq. (17) accounts for the shear effect

Reissner (1944) also pointed out that the shear contribution in this partial differential equation may be neglected if the Laplacian of the applied pressure is sufficiently small (the same remark holds for the Bresse-Timoshenko-Ehrenfest beam theory).

As analyzed by Batista (2010), in case of transversally inextensible plate (see also Kromm, 1953 for the introduction of transversally inextensible condition), Reissner deflection equation could be rewritten as:

$$
D \Delta^{2} v=p-\frac{h^{2}(2-\omega v)}{10(1-v)} \Delta p
$$

where $\omega$ is a dimensionless factor which is equal to 0 for transversally inextensible plate, and $\omega$ is equal to unity for isotropic plates. Reissner $(1944,1945)$ derived Eq. (15) which is Eq. (16) with $\omega=1$. Bolle (1947) obtained the deflection equation of a plate, equivalent in Eq. (16) with the transversal inextensibility $\omega=0$ (see Figure 10):

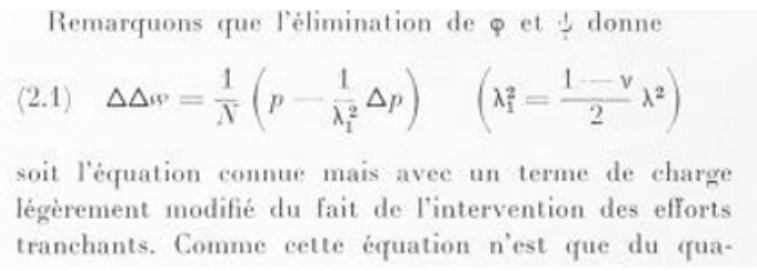

Figure 10 - Eq. (2.1) of Bolle (1947); With the notation of Bolle (1947), $N$ is equal to the bending stiffness $D$ used in this paper, and $\lambda^{2}=10 / h^{2}$ 
Eq. (2.1) of Bolle (1947) can be equivalently written by

$$
D \Delta^{2} v=p-\frac{h^{2}}{5(1-v)} \Delta p
$$

which can be seen as a kind of generalization of Bresse-Timoshenko-Ehrenfest beam theory for a plate:

$$
D \Delta^{2} v=p-\frac{D}{\kappa G h} \Delta p
$$

with a shear correction factor $\kappa=5 / 6$, as considered by Engesser (1891) and Föppl (1897) for statics of beams. Eq. (18) can be seen as the plate generalization of the governing equation Eq. (11) valid for the Bresse-Timoshenko-Ehrenfest beam. Furthermore, Bolle (1947) also first introduced a three-field kinematic theory (one deflection and two independent rotations), which is the natural generalization of Bresse-Timoshenko-Ehrenfest beam model at the plate level. As mentioned by Bolle (1947) - see also the extensive paper of Reissner (1985), the plate theory developed by Bolle and published in 1947 (Bolle, 1947) was already presented in September 1946 in Paris, during the sixth International Congress on Applied Mechanics. From an historical point of view, this would mean that Bolle (1946) was the first to have introduce a fully consistent shear plate theory based on a threefield kinematic field, as the natural plate extension of Bresse-Timoshenko-Ehhrenfest beam theory.

The bending moments are expressed by Bolle (1947) with respect to the rotations as (see Figure 11):

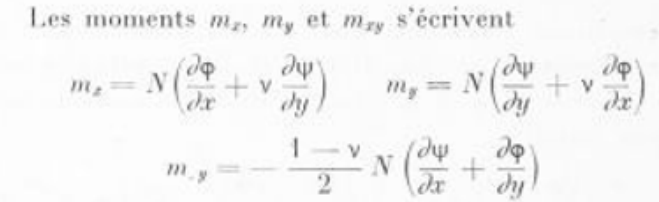

où $N$ désigne la rigidité de la plaque et $v$ le rapport de Poisson.

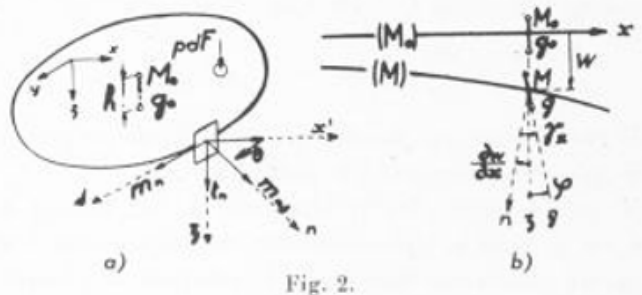

Figure 11 - Figure 2 of Bolle (1947) coupled with the bending constitutive law; Bolle's notation can be reexpressed with the notation of the paper $(\varphi, \psi, w)=\left(\psi_{x}, \psi_{y}, v\right)$ whereas the shear force is given by Bolle (1947) in Figure 12

$$
\begin{aligned}
& \text { Avec les formules et désignations } \\
& G=\frac{E}{2(1+v)} \quad N=\frac{E h^{3}}{12\left(1-v^{2}\right)} \quad \lambda^{2}=\frac{10}{h^{2}} \\
& \text { on peut écrire } \\
& t_{z}=\frac{(1-v) N}{2} \lambda^{2}\left(\varphi+\frac{\partial w}{\partial x}\right) \quad t_{g}=\frac{(1-v) N}{2} \lambda^{2}\left(\psi+\frac{\partial w}{\partial y}\right) .
\end{aligned}
$$

Figure 12 - Introduction of the shear force constitutive law in Bolle (1947); Bolle's notation can be reexpressed with the notation of the paper $(\varphi, \psi, w)=\left(\psi_{x}, \psi_{y}, v\right)$

The moment-curvature constitutive law is then expressed with the notation of this paper

$$
\left\{\begin{array}{c}
M_{x}=D\left(\frac{\partial \psi_{x}}{\partial x}+v \frac{\partial \psi_{y}}{\partial y}\right) \\
M_{y}=D\left(\frac{\partial \psi_{y}}{\partial y}+v \frac{\partial \psi_{x}}{\partial x}\right) \\
M_{x y}=\frac{D(1-v)}{2}\left(\frac{\partial \psi_{y}}{\partial x}+\frac{\partial \psi_{x}}{\partial y}\right)
\end{array}\right.
$$

and the shear force constitutive equation can be written as

$$
\left\{\begin{array}{l}
V_{x}=\kappa G h\left(\psi_{x}-\frac{\partial v}{\partial x}\right) \\
V_{y}=\kappa G h\left(\psi_{y}-\frac{\partial v}{\partial y}\right)
\end{array}\right.
$$

The equilibrium equations (including static distributed forces and inertia effects) can be written:

$$
\left\{\begin{array}{l}
\frac{\partial M_{x}}{\partial x}+\frac{\partial M_{x y}}{\partial y}-V_{x}=\frac{\rho h^{3}}{12} \frac{\partial^{2} \psi_{x}}{\partial t^{2}} \\
\frac{\partial M_{x y}}{\partial x}+\frac{\partial M_{y}}{\partial y}-V_{y}=\frac{\rho h^{3}}{12} \frac{\partial^{2} \psi_{y}}{\partial t^{2}} \\
\frac{\partial V_{x}}{\partial x}+\frac{\partial V_{y}}{\partial y}=-\rho h \frac{\partial^{2} v}{\partial t^{2}}+p
\end{array}\right.
$$

Coupling the equilibrium equations with the moment and shear force constitutive law leads in statics (neglecting inertia forces) to the partial differential equation for the deflection of Bolle (1947) Eq. (17) reexpressed with Eq. (18). Eq. (21) is a coupled system of partial differential equations which can be equivalently expressed in term of kinematic variables (see Uflyand, 1948 or Mindlin, 1951 for 
such a system with both static and dynamic contributions)

$$
\left\{\begin{array}{c}
\frac{D}{2}\left[(1-v) \Delta \psi_{x}+(1+v) \frac{\partial \phi}{\partial x}\right] \\
-\kappa G h\left(\psi_{x}-\frac{\partial v}{\partial x}\right)=\frac{\rho h^{3}}{12} \frac{\partial^{2} \psi_{x}}{\partial t^{2}} \\
\frac{D}{2}\left[(1-v) \Delta \psi_{y}+(1+v) \frac{\partial \phi}{\partial y}\right] \\
-\kappa G h\left(\psi_{y}-\frac{\partial v}{\partial y}\right)=\frac{\rho h^{3}}{12} \frac{\partial^{2} \psi_{y}}{\partial t^{2}} \\
\kappa G h(\phi-\Delta v)=-\rho h \frac{\partial^{2} v}{\partial t^{2}}+p \\
\text { with } \phi=\frac{\partial \psi_{x}}{\partial x}+\frac{\partial \psi_{y}}{\partial y}
\end{array}\right.
$$

Related works on shear plate theories are the works of Hencky (1947) who developed the same year, the bending equations of a plate with shear effect, based on a three-field kinematic theory (one deflection and two independent rotations):

$$
D \Delta^{2} v=p-\frac{h^{2}}{6(1-v)} \Delta p
$$

which can be seen as a kind of generalization of Bresse theory based on $\kappa=1$, at the plate scale (see Figure 13).

$$
\frac{E^{\prime} h^{3}}{12} \Delta \Delta \varphi=p-\frac{h^{2}}{6} \frac{m}{m-1} \Delta p
$$

Figure 13 - Eq. (III-c) of Hencky (1947); Hencky's notation can be reexpressed with the notation of the paper $\left(\varphi, E^{\prime}, m\right)=\left(v, \frac{E}{1-v^{2}}, \frac{1}{v}\right)-$ Generalization of Bresse's theory to plate

Uflyand (1948) derived the general static and dynamics plate equations of the so-called UflyandMindlin plate model (generalization of the beam equations Eq. (14) to the plate model, with a threefield kinematics):

$$
\begin{gathered}
D \Delta^{2} v-\frac{\rho h^{3}}{12}\left(1+\frac{12 D}{h^{3} \kappa G}\right) \frac{\partial^{2} \Delta v}{\partial t^{2}}+\rho h \frac{\partial^{2} v}{\partial t^{2}}+ \\
\frac{\rho^{2} h^{3}}{12 \kappa G} \frac{\partial^{4} v}{\partial t^{4}}=p-\frac{D}{\kappa G h} \Delta p+\frac{\rho h^{2}}{12 \kappa G} \frac{\partial^{2} p}{\partial t^{2}}
\end{gathered}
$$

Дифференцируя второе пз этих уравненпй по $x$, а третье по $y$, сқадывая результаты п исключая из всей системы комбннацию $\partial x_{z} / \partial x+\partial \alpha_{p} / \partial y$, получим уравненле поперечных колебапий пластины

$$
\begin{gathered}
\Delta \Delta w-\left(\frac{1}{v_{1}^{2}}+\frac{1}{v_{2}{ }^{2}}\right) \frac{\partial^{2} \Delta w}{\partial t^{2}}+\frac{1}{v_{1}{ }^{2} c_{2}{ }^{2}} \frac{\partial^{4} w}{\partial t^{4}}+V \frac{\partial^{2} w}{\partial t^{2}}=\frac{q}{D}+\frac{1}{k \mu h}\left(\frac{\rho J}{D} \frac{\partial^{2} q}{\partial t^{2}}-\Delta q\right) \\
\text { Здесь } \\
v_{1}=\sqrt{\frac{E}{\rho\left(1-v^{2}\right)}}, \quad v_{2}=\sqrt{\frac{2 \mu}{3 p}}, \quad V=\frac{\rho h}{D}
\end{gathered}
$$

Figure 14 - Eq. (2.7) of Uflyand (1948); Uflyand's notation can be reexpressed with the notation of the paper $(w, q)=(v, p)-$ Generalization of BresseTimoshenko-Ehrenfest's theory to plate (both in statics and in dynamics)

Eq. (24) is the generalization of Bresse-TimoshenkoEhrenfest's theory to plate (both in statics and in dynamics). Eq. (24) (or equivalently Eq. (2.7) of Uflyand, 1948) can be obtained from Eq. (22), which can be presented in the form of a coupled system of partial differential equation:

$$
\left\{\begin{array}{c}
\phi=\Delta v-\frac{\rho}{\kappa G} \frac{\partial^{2} v}{\partial t^{2}}+\frac{p}{\kappa G h} \\
\Delta v=\frac{\varrho h^{2}}{12 \kappa G} \frac{\partial^{2} \phi}{\partial t^{2}}+\phi-\frac{D}{\kappa G h} \Delta \phi
\end{array}\right.
$$

Eq. (24) (or equivalently Eq. (2.7) of Uflyand, 1948) is also the dynamics generalization of Eq. (18) derived in statics. Uflyand (1948) chose $\kappa=\frac{2}{3}$, as considered earlier by Timoshenko $(1916 ; 1921)$ for beam problems. Eq. (24) derived by Uflyand (1948) has been also confirmed later by Mindlin (1951), through variational arguments (see Figure 15).

$$
\begin{aligned}
\left(\nabla^{2}-\frac{\alpha_{\rho}}{G^{\prime}} \frac{\partial^{2}}{\partial t^{2}}\right)\left(D \nabla^{2}-\frac{\beta_{\rho} h^{3}}{12} \frac{\partial^{2}}{\partial t^{2}}\right) \bar{w}+\rho h \frac{\partial^{2} \bar{w}}{\partial t^{2}} \\
=\left(1-\frac{D \nabla^{2}}{G^{\prime} h}+\frac{\rho h^{2}}{12 G^{\prime}} \frac{\partial^{2}}{\partial t^{2}}\right) q \ldots \ldots[37]
\end{aligned}
$$

Equation [37] is the two-dimensional analog of Timoshenko's beam equation (6).

Figure 15 - Eq. (37) of Mindlin (1951); Mindlin's notation can be reexpressed with the notation of the paper $\left(w, q, G^{\prime}\right)=(v, p, \kappa G)-$ Generalization of Bresse-Timoshenko-Ehrenfest's theory to plate (both in statics and in dynamics) 
Mindlin (1951) used the energy formulation based on the strain energy density function

The analogous plate-strain-energy-function $\bar{W}$ is obtained by substituting Equations [10] into Equations [17] and integrating over the plate thickness, with the result

$$
\left.\begin{array}{rl}
2 \ddot{W}=M_{x} \frac{\partial \psi_{x}}{\partial x}+ & M_{y} \frac{\partial \psi_{y}}{\partial y}+M_{y x}\left(\frac{\partial \psi_{y}}{\partial x}+\frac{\partial \psi_{s}}{\partial y}\right) \\
& +Q_{x}\left(\frac{\partial \bar{w}}{\partial x}+\psi_{x}\right)+Q_{y}\left(\frac{\partial \bar{w}}{\partial y}+\psi_{v}\right)
\end{array}\right\} \ldots
$$

and this becomes, through Equations [5]

$4 \bar{W}=D(1+\mu)\left(\Gamma_{x}+\Gamma_{y}\right)^{2}+2 \kappa^{2} G h\left(\Gamma_{y z^{2}}+\Gamma_{s s^{2}}\right)$

$+D(1-\mu)\left[\left(\Gamma_{x}-\Gamma_{y}\right)^{2}+\Gamma_{y x}^{2}\right] \ldots \ldots[19]$

Figure 16 - Plate strain energy function from Mindlin (1951) with the notation of the paper $\left(w, \mu, \kappa^{2}\right)=(-v, v, \kappa)$

The strain energy density function $\bar{W}$ given by Eq. (19) of Mindlin (1951) can be rewritten with the notation of the paper:

$$
\begin{gathered}
\bar{W}=\frac{D}{2}\left(\Gamma_{x}^{2}+\Gamma_{y}^{2}+2 v \Gamma_{x} \Gamma_{y}\right)+\frac{D}{4}(1-v) \Gamma_{x y}^{2} \\
+\frac{\kappa G h}{2}\left(\Gamma_{x z}^{2}+\Gamma_{y z}^{2}\right)
\end{gathered}
$$

where the generalized strain (curvature and shear strain) are defined, according to Mindlin (1951) from

$$
\begin{gathered}
\Gamma_{x}=\frac{\partial \psi_{x}}{\partial x} ; \Gamma_{y}=\frac{\partial \psi_{y}}{\partial y} ; \Gamma_{x y}=\frac{\partial \psi_{y}}{\partial x}+\frac{\partial \psi_{x}}{\partial y} ; \\
\Gamma_{x z}=\psi_{x}-\frac{\partial v}{\partial x} \quad \text { and } \Gamma_{y z}=\psi_{y}-\frac{\partial v}{\partial y}
\end{gathered}
$$

The generalized stresses (bending moments and shear forces) may be deduced from derivation of this strain energy density function:

$$
\begin{gathered}
\mathrm{M}_{x}=\frac{\partial \bar{W}}{\partial \Gamma_{x}} ; \mathrm{M}_{y}=\frac{\partial \bar{W}}{\partial \Gamma_{y}} ; \mathrm{M}_{x y}=\frac{\partial \bar{W}}{\partial \Gamma_{x y}} ; \\
\mathrm{V}_{x}=\frac{\partial \bar{W}}{\partial \Gamma_{x z}} \text { and } \mathrm{V}_{y}=\frac{\partial \bar{W}}{\partial \Gamma_{y z}}
\end{gathered}
$$

This energy function Eq. (28) could be equivalently formulated in a stress-based framework, as used by Reissner $(1944 ; 1945)$ in the case of isotropic plates:

$$
\begin{aligned}
& \bar{W}^{*}=\frac{1}{2 D\left(1-v^{2}\right)}\left(\mathrm{M}_{x}^{2}+\mathrm{M}_{y}^{2}-2 v \mathrm{M}_{x} \mathrm{M}_{y}\right)+ \\
& \frac{1}{D(1-v)} \mathrm{M}_{x y}^{2}+\frac{1}{2 \kappa G h}\left(\mathrm{~V}_{x}^{2}+\mathrm{V}_{y}^{2}\right)
\end{aligned}
$$

The generalized strains can be deduced by derivation:

$$
\begin{gathered}
\Gamma_{x}=\frac{\partial \bar{W}^{*}}{\partial \mathrm{M}_{x}} ; \Gamma_{y}=\frac{\partial \bar{W}^{*}}{\partial \mathrm{M}_{y}} ; \Gamma_{x y}=\frac{\partial \bar{W}^{*}}{\partial \mathrm{M}_{x y}} ; \\
\Gamma_{x z}=\frac{\partial \bar{W}^{*}}{\partial \mathrm{v}_{x}} \text { and } \Gamma_{y z}=\frac{\partial \bar{W}^{*}}{\partial \mathrm{v}_{y}}
\end{gathered}
$$

Mindlin (1951) also calibrated the shear correction factor from the exact antisymmetric vibration mode of thickness-shear vibration and found:

$$
\kappa=\frac{\pi^{2}}{12}
$$

The calibration of the shear correction factor of the so-called Uflyand-Mindlin plate model from threedimensional elasticity has been performed analytically by Hutchinson (1984), Wittrick (1987), Stephen (1997) by comparing the natural frequencies of the three-dimensional solution with the one of the Uflyand-Mindlin plate model. They obtained the shear correction factor valid for plates:

$$
\kappa=\frac{5}{6-v}
$$

This shear correction factor may be also obtained from the paper of Timoshenko (1922) by considering the plane strain assumptions in Eq. (7)

$$
\begin{aligned}
& v=\frac{v^{\prime}}{1-v^{\prime}} \text { and } \quad \kappa=\frac{5(1+v)}{6+5 v} \\
& \kappa=\frac{5}{6-v^{\prime}}
\end{aligned}
$$

Such a correspondence between the shear correction factor of the beam model and the one of the plate model was also observed by Stephen (1997). Hutchinson (1984) obtained this shear correction factor from the vibration analysis of a circular plate, Wittrick (1987) considered the free vibration of a simply supported rectangular plate and Stephen (1997) derived this value from plane strain exact Rayleigh-Lamb frequency equation for infinite plate.

This shear correction factor was also numerically obtained by comparison of the three-dimensional elasticity solution with Uflyand-Mindlin plate model by Srinivas et al (1970) or Dawe (1979) who obtained a shear correction factor $\kappa \approx 0.88$ for $v=$ 0.3 , which is exactly the value given by the formulae Eq. (32).

Goldenveizer et al (1990, 1993) used an asymptotic expansion of the mixed displacement and stress field with respect to some small parameters linked to the relative depth of the plates (or shells) with shear effects (see also Kaplunov et al, 1998). They also obtained the shear correction factor given by Eq. (32). More recently, Elishakoff et al (2017) showed 
from an asymptotic expansion of the displacement field (solely) that the asymptotically-based shear correction factor is the one given by Eq. (32). They also derived a truncated Uflyand-Mindlin plate model issued of the three-dimensional elasticity governing equations:

$$
D \Delta^{2} v-\frac{\rho h^{3}}{12}\left(1+\frac{12 D}{h^{3} \kappa G}\right) \frac{\partial^{2} \Delta v}{\partial t^{2}}+\rho h \frac{\partial^{2} v}{\partial t^{2}}=0
$$

This truncated Uflyand-Mindlin plate equation has been also obtained by Goldenveizer et al (1990, 1993), Elishakoff (1994), Kaplunov et al (1998) and Mindlin (2006) (based on a monograph written by Midlin in 1955 - Mindlin, 1955), also using asymptotic expansion considerations. Eq. (34) is the plate analogy of the truncated Bresse-TimoshenkoEhrenfest equation derived from asymptotic arguments.

Finally, it is worth mentioning that the bending constitutive law Eq. (19) expressed with the curvature variables may be reexpressed using the displacement and the shear forces, as detailed in Figure 17 from the paper of Reissner (1944):

$$
\begin{array}{ll}
M_{x}-\frac{h^{2}}{5(1-\nu)}\left(\frac{\partial V_{z}}{\partial x}+\nu \frac{\partial V_{y}}{\partial y}\right)-\frac{h^{2} \nu}{10(1-\nu)} p=-D\left(\frac{\partial^{2} w}{\partial x^{2}}+\nu \frac{\partial^{2} w}{\partial y^{2}}\right) & \text { (15a) } \\
M_{y}-\frac{h^{2}}{5(1-\nu)}\left(\frac{\partial V_{y}}{\partial y}+\nu \frac{\partial V_{z}}{\partial x}\right)-\frac{h^{2} v}{10(1-\nu)} p=-D\left(\frac{\partial^{2} w}{\partial y^{2}}+\nu \frac{\partial^{2} w}{\partial x^{2}}\right) & \text { (15b) } \\
H-\frac{h^{2}}{10}\left(\frac{\partial V_{z}}{\partial y}+\frac{\partial V_{y}}{\partial x}\right)=-(1-\nu) D \frac{\partial^{2} w}{\partial x \partial y} & \text { (15e) }
\end{array}
$$

Figure 17 - Reformulation of Reissner model in term of single displacement variable (from Reissner, 1944)

In fact, by coupling Eq. (19) and Eq. (20), the bending constitutive law can be reformulated as:

$$
\left\{\begin{array}{c}
M_{x}-\frac{D}{\kappa G h}\left(\frac{\partial V_{x}}{\partial x}+v \frac{\partial V_{y}}{\partial y}\right)=D\left(\frac{\partial^{2} v}{\partial x^{2}}+v \frac{\partial^{2} v}{\partial y^{2}}\right) \\
M_{y}-\frac{D}{\kappa G h}\left(\frac{\partial V_{y}}{\partial y}+v \frac{\partial V_{x}}{\partial x}\right)=D\left(\frac{\partial^{2} v}{\partial y^{2}}+v \frac{\partial^{2} v}{\partial x^{2}}\right) \\
M_{x y}-\frac{D(1-v)}{2 \kappa G h}\left(\frac{\partial V_{y}}{\partial x}+v \frac{\partial V_{x}}{\partial y}\right)=D(1-v) \frac{\partial^{2} v}{\partial x \partial y}
\end{array}\right.
$$

In the static range, according to Eq. (21), the shear forces may be simply expressed in term of bending moments:

$$
V_{x}=\frac{\partial M_{x}}{\partial x}+\frac{\partial M_{x y}}{\partial y} \text { and } V_{y}=\frac{\partial M_{x y}}{\partial x}+\frac{\partial M_{y}}{\partial y}
$$

which means that the bending-curvature constitutive law in the static range may be expressed in an implicit differential form:

$$
\left\{\begin{array}{c}
M_{x}-\frac{D}{\kappa G h}\left[\frac{\partial^{2} M_{x}}{\partial x^{2}}+(1+v) \frac{\partial^{2} M_{x y}}{\partial x \partial y}+v \frac{\partial^{2} M_{y}}{\partial y^{2}}\right] \\
=D\left(\frac{\partial^{2} v}{\partial x^{2}}+v \frac{\partial^{2} v}{\partial y^{2}}\right) \\
M_{y}-\frac{D}{\kappa G h}\left[\frac{\partial^{2} M_{y}}{\partial y^{2}}+(1+v) \frac{\partial^{2} M_{x y}}{\partial x \partial y}+v \frac{\partial^{2} M_{x}}{\partial x^{2}}\right] \\
=D\left(\frac{\partial^{2} v}{\partial y^{2}}+v \frac{\partial^{2} v}{\partial x^{2}}\right) \\
M_{x y}-\frac{D(1-v)}{2 \kappa G h}\left[\frac{\partial^{2} M_{x}}{\partial x \partial y}+\frac{\partial^{2} M_{y}}{\partial x \partial y}+\frac{\partial^{2} M_{x y}}{\partial x^{2}}+\frac{\partial^{2} M_{x y}}{\partial y^{2}}\right] \\
=D(1-v) \frac{\partial^{2} v}{\partial x \partial y}
\end{array}\right.
$$

One may recognize an implicit differential form of a nonlocal Germain-Lagrange plate model, where the length scale factor is controlled by the ratio between the bending stiffness and the shear stiffness. In the limit, for an infinite shear stiffness, i.e. for $D / \kappa G h \rightarrow 0$, the local Germain-Lagrange plate model (see Bucciarelli and Dworsky, 1980 for a detailed presentation of the contribution of Sophie Germain in the derivation of thin plate theory during the XIXth century - see also Timoshenko, 1953 or Truesdell, 1991 or Kawano, 2013), also referred as Kirchhoff-Love plate model, is asymptotically obtained:

$$
\left\{\begin{array}{l}
M_{x}=D\left(\frac{\partial^{2} v}{\partial x^{2}}+v \frac{\partial^{2} v}{\partial y^{2}}\right) \\
M_{y}=D\left(\frac{\partial^{2} v}{\partial y^{2}}+v \frac{\partial^{2} v}{\partial x^{2}}\right) \\
M_{x y}=D(1-v) \frac{\partial^{2} v}{\partial x \partial y}
\end{array}\right.
$$

The reformulation of Bresse-Timoshenko-Ehrenfest theory in a nonlocal Euler-Bernoulli beam theory was already recognized by Challamel (2011) for buckling applications. In fact, the BresseTimoshenko-Ehrenfest beam equation analogous to Eq. (35) is expressed by

$$
M-\frac{E I}{\kappa G A} V^{\prime}=E I v^{\prime \prime}
$$

In the static range according to Eq. (1), $V=M^{\prime}$ so that one recognizes a nonlocal differential EulerBernoulli beam theory: 


$$
M-\frac{E I}{\kappa G A} M^{\prime \prime}=E I v^{\prime \prime}
$$

where the differential equation has been used by Eringen (1983) for introducing length scales in nonlocal elasticity. Bresse-Timoshenko-Ehrenfest theory is a nonlocal Euler-Bernoulli beam theory where the length scale is introduced from the ratio between the bending stiffness and the shear stiffness $E I / \kappa G A$.

In the limit, for an infinite shear stiffness, i.e. for $E I / \kappa G A \rightarrow 0$, the local Bernoulli-Euler beam model (see Timoshenko, 1953 for an historical presentation of Euler and Bernoulli on thin beam theory during the XVIII ${ }^{\text {th }}$ century) is asymptotically obtained:

$$
M=E I v^{\prime \prime}
$$

\section{Conclusions}

Whereas Bresse-Timoshenko-Ehrenfest equations have been elaborated mainly during more than half a century between 1859 and 1922 (Bresse defined the two-field beam kinematics and the calibration of the shear correction factor has been finalized by Timoshenko in 1922, even if the basics results were available in his letter dated from 1913, in collaboration with Ehrenfest), the Uflyand-Mindlin plate theory has been built in a more compact period, mainly between 1944 and 1951 .

Reissner (1944, 1945) first developed a static (or stress-based) formulation, but the kinematic theory of Uflyand-Mindlin plate model (or Bolle-UflyandMindlin plate model) has been first elaborated by Bolle (1947) and Hencky (1947) for static loading, before the full generalization to dynamics by Uflyand one year later, in 1948, and its complete variational derivation by Mindlin (1951). A lot of efforts were spent since almost half of the century to calibrate the shear correction factor and to enrich the kinematics of Uflyand-Mindlin plate theory. It is quite surprising that the shear correction factors of both the Bresse-Timoshenko-Ehrenfest beam model and its Uflyand-Mindlin plate analogy were already implicitly available in the paper of Timoshenko (1922).

\section{References}

Abramovich H. and Elishakoff I., Application of the Krein's method for determination of natural frequencies of periodically supported beam based on simplified Bresse Timoshenko equations, Acta Mechanica, 66, 39 59, 1987.

Artobolevsky I.I., Bobrovnitsky Yu. I and Genkin M.D., Introduction to Acoustic Dynamics of Machines, Moscow: "Nauka" Publishing House, 1979 (in Russian).

Barré de Saint-Venant A., Mémoire sur la flexion des prismes, sur les glissements transversaux et longitudinaux qui l'accompagnent lorsqu'elle ne s'opère pas uniformément ou en arc de cercle, et sur la forme courbe affectée alors par leurs sections transversales primitivement planes., J. Math. Pures et Appliquées, 2ème Série, 1, 89-189, 1856.

Batista M., An elementary derivation of basic equations of the Reissner and Mindlin plate theories, Engineering Structures, 32, 906-909, 2010.

Berdichevskii, V. L., Dynamic theory of thin elastic plates, Izv. AN SSSR Mekhanika Tverdogo Tela 8(6), 99-109, 1973.

Berdichevskii V.L. and Starosel'skii L.A., On the theory of curvilinear Timoshenko-type rods, P.M.M. USRR, 47, 6, 809-817, 1983.

Bert C.W., Nonprismatic Shear Beams, Journal of Structural Engineering, 111, 1167-1168, 1985.

Bolle L. Contribution au problème linéaire de flexion d'une plaque élastique, Bull Tech De La Suisse Romande, 73(21), 281-285, 1947.

Borg S.F. and Gennaro J.J., Modern structural analysis, Van Nostrand Reinhold, New York, 1969.

Bresse J.A.C., Cours de mécanique appliquée Résistance des matériaux et stabilité des constructions, Paris, Gauthier-Villars, 1859 (in French).

Bucciarelli L.L. and Dworsky N., Sophie Germain An essay in the history of the theory of elasticity, D. Reidel Publishing Company, 1980.

Carrera E., Giunta G. and Petrolo M., Beam structures - Classical and advanced theories, Wiley, 2011.

Challamel N., Higher-order shear beam theories and enriched continuum, Mechanics Research Communications, 38, 388-392, 2011. 
Dawe D.J., Finite strip models for vibration of Mindlin plates, J. Sound Vibration, 59, 3, 441-452, 1979.

Elishakoff I. and Lubliner E., Random vibration of a structure via classical and nonclassical theories, in: Probabilistic Methods in the Mechanics of Solids and Structures (S. Eggwertz and N.C. Lind, eds.), Berlin: Springer, 455-467, 1985.

Elishakoff I. and Irretier H., Refined dynamical theories of beams, plates and shells and their applications, Proceedings of the Euromech Colloquium 219, Springer-Verlag, Berlin, 1987.

Elishakoff I. and Livshits D., Some closed form solutions in random vibrations of Timoshenko beams, Journal of Probabilistic Engineering Mechanics, 4, 49 54, 1989.

Elishakoff I., Generalization of the Bolotin's dynamic edge-effect method for vibration analysis of Mindlin plates, in Proceedings of the 1994 National Conference on Noise Control Engineering (J.M. Cuschieri, S.A.L. Glegg and D.M. Yeager, eds.), pp. 911-916, New York, 1994.

Elishakoff I., An equation both more consistent and simpler than Bresse-Timoshenko equation, in Advances in Mathematical Modeling and Experimental Methods for Materials and Structures (R. Gilat and L. Sills-Banks, eds.), 249-254, Springer Verlag, Berlin 2010.

Elishakoff I., Kaplunov J. and Nolde E., Celebrating the centenary of Timoshenko's study of effects of shear deformation and rotary inertia. Appl. Mech. Rev 67(6), 2015.

Elishakoff I., Hache F. and Challamel N., Vibrations of asymptotically and variationally based UflyandMindlin plate models, Int. J. Eng. Sc., 116, 58-73, 2017.

Elishakoff I., Handbook on Timoshenko-Ehrenfest and Uflyand-Mindlin plate theories, World Scientific Publishing Company, 2019-a.

Elishakoff I., Stepan Prokofievich Timoshenko and America, Zeitschrift für Angewandte Mathematik und Mechanik, 99, 3, e201800338, 2019-b.

Elishakoff I., J.P. Den Hartog about S.P. Timoshenko: fifty years later, Mathematics and Mechanics of Solids, 24, 5, 1340-1348, 2019-c.

Engesser F., Die Knickfestigkeit gerader Stäbe, Centralblatt der Bauverwaltung, 11, 49, 483-486, 1891 (in German).
Eringen A.C., On differential equations of nonlocal elasticity and solutions of screw dislocation and surface waves, J. Appl. Phys., 54, 4703-4710, 1983.

Föppl A., Vorlesungen über Technische Mechanik Dritter Band, Festigkeitslehre, Lepizig, 1897 (in German).

Goldenveizer AL, Kaplunov JD, Nolde EV. Asymptotic analysis and refinement of TimoshenkoReisner-type theories of plates and shells, Trans. Acad. Sci. USSR. Mekhanika Tverd. Tela, 1990.

Goldenveizer A.L., Kaplunov J.D. and Nolde E.V., On Timoshenko-Reissner type-theories of plates and shells, Int. J. Solids Structures, 30, 5, 675-694, 1993.

Hencky H., Über die Berücksichtigung der Schubverzerrung in ebenen Platten, Ingenieur Archiv., 16, 1, 72-76, 1947 (in German).

Henrych J., The Dynamics of arches and frames, Elsevier, Amsterdam, 1981.

Hutchinson J.R., Vibrations of thick free circular plates, exact versus approximate solutions, J. Appl. Mech., 51, 581-585, 1984.

Irie T., Yamada G. and Tanaka K., Natural frequencies of in-plane vibration of arcs, J. Appl. Mech., 50, 449-452, 1983.

Issa M.S., Wang T.M. and Hsiao B.T., Extensional vibrations of continuous circular curved beams with rotary inertia and shear deformation, I: free Vibration, J. Sound Vibration, 114, 297-308, 1987.

Kaneko T., On Timoshenko's correction for shear in vibrating beams, J. Physics D : Applied Physics, 8, 1927-1939, 1975.

Kang K., Bert C.W. and Striz A.G., Vibration analysis of shear deformable circular arches by the differential quadratic method, J. Sound Vibration, 181, 353-360, 1995.

Kaplunov J.D., Kossovich L.Y. and Nolde E.V., Dynamics of thin walled elastic bodies, Academic Press, 1998.

Kawano A., A uniqueness theorem for the determination of sources in the Germain-Lagrange plate equation, J. Math. Anal. Appl., 402, 191-200, 2013.

Kromm A., Verallgemeinerte Theorie der Plattenstatik, Ingenieur-Archiv, 21, 266-86, 1953. 
Lamb H., On waves in an elastic plate, Proc. R. Soc. London, Ser. A, 93, 114-128, 1917.

Luongo A. and Zulli D., Mathematical models of beams and cables, ISTE-Wiley, 2013.

Mindlin R.D., Influence of rotary inertia and shear on flexural motions of isotropic elastic plates, $J$. Appl. Mech., 18, 31_38, 1951.

Mindlin R.D. and Deresiewicz H., Timoshenko's shear coefficient for flexural vibration of beams, in: Proceedings of the 2nd U.S. National Congress in Applied Mechanics, 175-178, New York, 1955.

Mindlin R.D., An introduction to the mathematical theory of vibrations of elastic plates, A monograph prepared for U. S. Army Signal Corps Engineering Laboratories, Fort Monmouth, N. J., Department of the Army Project 3-99-11-022, Signal Corps Project 142B, Signal Corps Contract DA-36-039 SC-56772, 1955.

Mindlin R.D. (J. Yang, ed.), An introduction to the mathematical theory of vibration of elastic plates, World Scientific, Singapore, 2006.

Morley L.S.D., Elastic waves in a naturally curved rod, Quart. J. Mech. Appl Math., 14, 155-172, 1961.

Rao S.S. and Sundararajan V., In-plane flexural vibrations of circular rings, Journal of Applied Mechanics, 36, 3, 620-625, 1969.

Rao S.S., Vibration of continuous systems, John Wiley \& Sons, 2007.

Rayleigh Lord, On the free vibration of an infinite plate of homogeneous isotropic elastic matter, Proc. London Math. Soc., 20, 225-234, 1889.

Reddy J.N., Mechanics of laminated composite plates and shells - Theory and analysis, 2nd edn. CRC Press, Boca Raton, Florida, 2004.

Reissner E., On the theory of bending of elastic plates, Studies in Applied mathematics., 23, 1-4, 184-191, 1944.

Reissner E., The effect of transverse shear deformation on the bending of elastic plates, ASME Journal of Applied Mechanics, 12, 69-77, 1945.

Reissner E., On transverse bending of plates including the effect of transverse shear deformation, Int. J. Solids Structures, 11, 569-573, 1975.

Reissner E., Reflections on the theory of elastic plates, Applied Mechanics Reviews, 38, 1453-1464, 1985.
Rubin M.B., On the quest for the best Timoshenko shear coefficient, J. Appl. Mech., 70, 154-157, 2003.

Srinivas S., Joga Rao C.V. and Rao A.K., An exact analysis for vibration of simply-supported homogeneous and laminated thick rectangular plates, J. Sound Vib., 12, 2, 187-199, 1970.

Stephen N.G., Considerations on second order beam theories, Int. J. Solids Structures, 17, 325-333, 1981.

Stephen N.G., Mindlin plate theory: best shear coefficient and higher spectra validity, J. Sound Vibration, 202, 4, 539-553, 1997.

Timoshenko S.P., Letter addressed to P. Ehrenfest, January 10 1913, (in Russian), 1913 (see Elishakoff, 2019).

Timoshenko S.P., A Course in theory of elasticity, Petrograd, (in Russian), 1916.

Timoshenko S.P., On the differential equation for the flexural vibrations of prismatical rods, Glasnik Hrvatskoga Prirodoslovnoga Društva (Herald of the Croatian Nature Association), 32, 2, 55-57, Zagreb, 1920.

Timoshenko S.P., On the correction for shear of the differential equation for transverse vibrations of prismatic bars, Philosophical Magazine, 41, 744746, 1921.

Timoshenko S.P., On the transverse vibration of bars with uniform cross-section, Philosophical Magazine, 43, 125-131, 1922.

Timoshenko S.P., Strength of materials - Part 1 Elementary theory and problems, Van Nostrand Company, New-York, $1^{\text {st }}$ Edition, 1930, $2^{\text {nd }}$ edition, 1940 .

Timoshenko S.P., History of strength of materials with a brief account of the history of theory of elasticity and theory of structures, Mc Graw-Hill Book Company, New-York, 1953.

Timoshenko S.P. and Woinowsky-Krieger S., Theory of plates and shells, New York: McGrawHill, Second Edition, 1959.

Truesdell C., Sophie Germain: Fame earned by stubborn error, Bollettino di Storia delle Scienze Matematiche, XI, 2, 3-24, 1991.

Uflyand Y.S., The propagation of waves in the transverse vibrations of bars and plates, Prikl. Math. Mech., 12, 287-461, 1948. 
Wang C.M., Reddy J.N. and Lee K.H., Shear deformable beams and plates: Relationships with classical solutions, Elsevier, Oxford, UK, 2000.

Wittrick W.H., Analytical, three-dimensional elasticity solutions to some plate problems, and some observations on Mindlin's plate theory, Int. J. Solids Structures, 23, 441-464, 1987. 